\title{
Temporal evolution of vorticity staircases in randomly strained two-dimensional vortices
}

\author{
by M. R. Turner ${ }^{1}$ \\ Department of Mathematics, University of Surrey, \\ Guildford, Surrey GU2 $7 X H$, England
}

- Abstract -

The evolution of a Gaussian vortex subject to a weak-external-random $n$ fold multipolar strain field is examined using fully nonlinear simulations. The simulations show that at large Reynolds numbers, fine scale steps form at the periphery of the vortex, before merging, generally leaving one large step, which acts as a barrier between the vorticity within the coherent core and the surrounding, well mixed, 'surf zone'.

It is shown for $n=2$ that the width and the number of fine scale steps which initially form at the periphery of the vortex is dependent on the strain parameters, but that the range of radial values for which steps initially occur is only dependent on $n$ and the amplitude of the strain field. A criteria is developed which can predict this range of radial values using the linear stability results of Le Dizès (J. Fluid Mech., vol. 406, 2000, p.175). This criteria is based upon the perturbation vorticity needing to be larger than some fraction of the vorticity gradient to flatten the vortex profile.

For $n=3$ and 4 , the radial step range is again predicted, and it is observed that for these higher wavenumbers the long lasting steps are narrower than the $n=2$ case. For $n=4$ the steps which form are so narrow that they do not persist very long before they are destroyed by the strain field and viscosity.

- November 5, 2014-

\section{Introduction}

The literature available on the motion of passive scalars in fluid flows is expansive. Once the motion of the fluid is determined (either kinematically or dynamically), the motion of the passive scalar added to the flow is simply found by advection. However, the study of

\footnotetext{
${ }^{1}$ m.turner@surrey.ac.uk
} 
flows containing a dynamic scalar which feeds back to modify the flow field are less well understood. In this paper we consider flows within two-dimensional coherent vortices, where vorticity plays the role of a dynamic scalar. In such flows, vorticity is mixed by the flow, but also feeds back to modify the flow itself. It is this dynamic feedback which leads to interesting evolutions of the fluid system.

In this work a coherent vortex is considered, which is placed in a weak external strain field to mimic the flow generated by the interaction with other distant vortices in the flow, or the motion of solid boundaries. This type of study has physical relevance to flows such as geophysical flows where vortices are associated with planetary rotations, and may be concentrated by antecedent diabatic processes $[1,2]$, and in turbulent flows where there are many vortices interacting with one another $[3,4,5]$. When an inviscid, axisymmetric coherent vortex, such as a Gaussian vortex, is placed in an instantaneous external strain field that acquires $n=2$ symmetry when viewed in the circular frame of reference, then the vortex is initially forced into an elliptical shape which generates an $n=2$ mode in the vorticity field of the vortex. This mode then evolves in time, dynamically feeding back on the core of the vortex, forcing the vortex to return to an axisymmetric state and spirally winding up the additional vorticity $[5,6]$. This dynamical feedback was further studied by Schecter et al. (2000) [7] and Balmforth et al. (2001) [8], with the latter constructing an asymptotic analysis linking the suppression of vorticity to the existence of a "quasimode' in the vortex. A quasi-mode is a damped mode of a flow which incorporates both neutral modes as well as contributions from the continuous spectrum. The decay rate of the quasi-mode can be linked to the value of a Landau pole of the flow field, which was first studied by Briggs et al. (1970) [9].

If instead the coherent vortex is placed in a weak-external strain field, whose axes are rotating with constant angular velocity $\alpha_{\text {ext }}$ for a fixed period of time, a critical layer occurs within the vortex at a radius $r_{\text {ext }}$ where the angular velocity of the vortex equals $\alpha_{\text {ext }}[10,11,12]$. The nature of the critical layer can be nonlinear, viscous or a combination of both by considering the relevant higher-order terms neglected by linear analysis $[13,10,11]$. Irrespective of the nature of the critical layer, Le Dizès (2000) [14] identified that the amplitude of the non-axisymmetric quasi-mode generated by the feedback process depends upon the value of $\alpha_{\text {ext }}$. The consequence of generating large non-axisymmetric quasi-modes in the vortex is that, via the nonlinear terms in the NavierStokes equations, these modes will feedback to modify the axisymmetric profile which changes the response of the vortex to future strains $[8,15,16]$.

If now the strain field is considered to be random, and applied continuously to the vortex, then Turner et al. (2009) [17] showed that the dynamic feedback from the vortex acts to generate fine scale steps in the periphery of the axisymmetric vortex. These steps eventually evolve to form a vorticity staircase. In the flat regions of the staircase the diffusivity is large, while the steep vorticity gradients in between act as barriers, preventing the transport of vorticity out of the vortex. They also observed the merger of steps overtime, typically leading to one or two large persistent steps, as well as the steps slowly drifting towards the core of the vortex. The evolution of the steps was followed in time using a weakly nonlinear expansion about the small strain amplitude. This led to a linear quasi-mode response equation, identical to that given in Le Dizès (2000) [14], feeding back on the axisymmetric vortex profile via a diffusion equation which evolves on a long time scale. The work presented in Turner et al. (2009) [17] was essentially an extension of the weakly nonlinear initial value problem considered in Bassom \& Gilbert 
(1999) [6] which forced a Gaussian vortex with a random strain field explicitly. However, due to the weakly nonlinear formulation, the simulations could not follow the vortex evolution very far in time due to the neglected higher order Fourier modes becoming significant. Turner et al. (2009) [17] resolved this issue by using the diffusion equation approach, but the neglected higher order modes of their work still needed to artificially suppressed, which was achieved by introducing a smoothing parameter.

The purpose of the current paper is two fold: Firstly by considering fully nonlinear solutions of the Navier-Stokes equations we are able to follow an explicitly forced Gaussian vortex for large times without the need to introduce a smoothing parameter, hence this work enhances the results presented in Turner et al. (2009) [17]. Secondly by using the linear feedback theory of Le Dizès (2000) [14] a criteria which predicts the range of radii over which steps are observed forming in the vortex is formulated. Using this linear feedback theory we are also able to highlight why the steps observed in Turner et al. (2009) [17], and the current paper drift towards to the centre of the vortex over time.

The formation of vorticity staircases also occurs in other flows, most notably in the jet structures which emerge in the oceans, our atmosphere and in the atmosphere of the gas giant planets such as Jupiter [18, 19, 20, 21]. The underlying process leading to the formation of vorticity staircases in these flows is identical to that studied in this article. A gradient of potential vorticity in the flow is homogenised in regions due to an external forcing which eventually leads to jets forming. The sharp vorticity gradients between the steps again act as barriers in the flow preventing the transport of vorticity.

The layout of the paper is as follows. In $\S 2$ we formulate the governing nonlinear equations and discuss the numerical scheme used in the simulations. In $\S 3$ we discuss the quasi-mode feedback process in more detail and highlight the strain field angular velocities where we expect to see a large feedback to the azimuthally averaged profile leading to the formation of vorticity staircases. In $\S 4$ we present the results of the simulations for strain fields with azimuthal wavenumbers $n=2,3$ and 4 , while concluding remarks and discussions are given in $\S 5$.

\section{Formulation}

We consider the evolution of a two-dimensional vortex with vorticity field $\omega$ and stream function $\psi$, which is placed in a weak, horizontally non-divergent, externally imposed, irrotational flow field given by the stream function $\psi_{\text {ext }}$. The evolution of this vortex is governed by the dimensionless vorticity equation

$$
\partial_{t} \omega-J\left(\psi+\psi_{\text {ext }}, \omega\right)=R^{-1} \nabla^{2} \omega, \quad \nabla^{2} \psi=-\omega, \quad \nabla^{2} \psi_{\text {ext }}=0,
$$

where $\mathbf{u}=\left(r^{-1} \partial_{\theta} \psi,-\partial_{r} \psi\right)$ in plane polar coordinates, and $J(a, b)=r^{-1}\left(\partial_{r} a \partial_{\theta} b-\partial_{\theta} a \partial_{r} b\right)$. The variables are non-dimensionalised by the total circulation of the vortex, $\Gamma$, so that the Reynolds number is defined by $R=\Gamma / \nu$, where $\nu$ is the kinematic viscosity. We consider the vortex to be in an unbounded domain, thus the stream function $\psi$ is not permitted to grow faster than $\log r$ at large values of $r$, while the external forcing can increase in powers of $r$. We are ultimately interested in results in the large Reynolds number limit, but in this work we present long time simulations so it is inevitable that the results will display some dependence on the value of the Reynolds number. For example, the destruction of fine scale structures in the vortex will occur on the shear-diffuse time scale of $O\left(R^{1 / 3}\right)[22,23,24,25]$ and the vortex will spread on the $O(R)$ time scale. 
The external flow field is chosen to represent an irrotational strain field of the form

$$
\psi_{\text {ext }}(r, \theta, t)=q(t) r^{n} e^{i n \theta}+\text { complex conjugate, }
$$

where $q(t)$ is the time dependent strength and orientation of the strain field, to be stipulated. This is a multipolar strain field with $n$-fold symmetry in $\theta$, where $n$ takes integer values. In the results which are presented in this paper we only consider strains with $n>1$, because $n=1$ is a pure translation mode and, thus is of no dynamical interest if $q(t)$ is independent of time.

Initially the vortex is axisymmetric $\omega(r, t=0)=\Omega_{0}(r), \psi(r, t=0)=\Psi_{0}(r)$ which is taken to be a Gaussian vortex given by

$$
\Omega_{0}(r)=\frac{1}{4 \pi} e^{-r^{2} / 4}, \quad \Psi_{0}(r)=-\frac{1}{2 \pi} \int_{\infty}^{r} \frac{1}{r}\left(e^{-r^{2} / 4}-1\right) d r .
$$

The angular velocity of this vortex as a function of $r$ is given by

$$
\alpha_{0}(r)=-\frac{1}{r} \frac{\partial \Psi_{0}}{\partial r}=\frac{1}{r^{2}} \int_{0}^{r} s \Omega_{0}(s) d s=\frac{1}{2 \pi r^{2}}\left(1-e^{-r^{2} / 4}\right)
$$

The initial axisymmetric vortex (2.3) is placed in the external strain field (2.2), which is switched on for $t>0$. The strain field forces the vortex into a non-axisymmetric shape by inducing higher order Fourier modes into the solution. The time evolution of the vorticity $\omega$ and stream function $\psi$ are found by time stepping (2.1) with a time step $\Delta t$, and seeking a solution for these quantities in the form of truncated Fourier series

$$
\omega(r, \theta, t)=\sum_{k=-N}^{N} \omega_{n k}(r, t) e^{\mathrm{i} n k \theta}, \quad \psi(r, \theta, t)=\sum_{k=-N}^{N} \psi_{n k}(r, t) e^{\mathrm{i} n k \theta} .
$$

The external strain field drives the $n^{\text {th }}$ mode of the vorticity field, which then excites higher Fourier modes through the non-linear terms $J\left(\psi+\psi_{\text {ext }}, \omega\right)$, as well as dynamically feeding back to modify the basic vorticity profile $\omega_{0}$. Equation (2.1) is integrated using a Crank-Nicholson scheme in spectral space on the linear terms while the nonlinear terms are updated in real space using the fifth order Adam-Bashforth method. The discretization in the radial direction uses central finite differences with $M=1500$ grid points in the range $0 \leq r \leq 15$, with the $\theta$ direction discretized using $N=128$ Fourier harmonics. The Poisson equation for $\psi$ in (2.1) is solved by inverting a tridiagonal matrix system via an LAPACK routine. The code is initially tested with $q(t)=0$ to check that the viscous decay of the vortex is captured correctly for the length of the runs in $\S 4$. The nonlinear runs are then checked so that they are independent of $\Delta t, N$ and $M$ for all times. The time step used in the simulations is $\Delta t=0.01$.

Before presenting the results of the numerical simulations we first discuss the significance of the critical layers generated in the vortex by the external strain field, and highlight the quasi-mode's feedback from these critical layers onto the azimuthally averaged vorticity profile $\omega_{0}(r, t)$. 


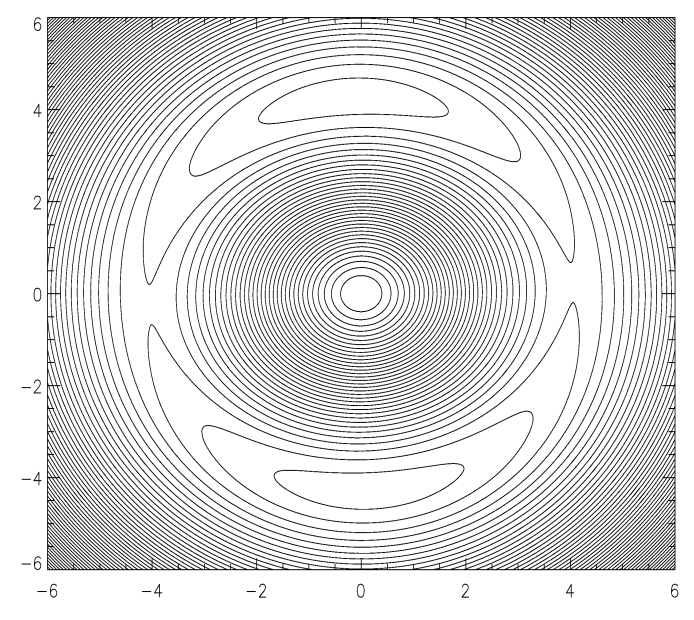

Figure 1: Streamlines showing a cat's eye structure in $\psi_{\text {co }}$ in the rotating frame of the imposed strain field with $n=2$.

\section{Critical radii and feedback responses}

For a two-dimensional vortex with an azimuthally averaged vorticity profile $\omega_{0}(r, t)$, its angular velocity is given by

$$
\alpha(r, t)=\frac{1}{r^{2}} \int_{0}^{r} \omega_{0}(s, t) s d s
$$

which for the Gaussian vortex (2.3) is given in (2.4). When this vortex is placed in the external strain field (2.2), with an amplitude $\widehat{A}$ and with axes which rotate with angular velocity $\alpha_{\text {ext }}$, i.e.

$$
q(t)=\widehat{A} \exp \left(-\mathrm{i} n \alpha_{\mathrm{ext}} t\right),
$$

then there exists a critical radius in the vortex where the angular velocity of the strain equals that of the vortex. Turner \& Gilbert (2007) [15] show that the co-rotation of the vortex and strain field leads to finite thickness cat's eyes being formed at the critical radius $r_{\text {ext }}$ where $\alpha\left(r_{\text {ext }}\right)=\alpha_{\text {ext }}$ (provided a solution of this equation exists). This cat's eye structure is formed initially in the co-rotating stream function

$$
\psi_{\mathrm{co}}(r, \theta, t)=\psi(r, \theta, t)+\frac{1}{2} \alpha_{\mathrm{ext}} r^{2}
$$

but by advection, it manifests itself as a cat's eye structure in the vorticity field too [16]. The streamlines for the cat's eye structure in the Gaussian vortex (2.3) is given in figure 1. Note that we choose the strain field to rotate in the same direction as the vortex otherwise no critical layer is generated, and the problem is uninteresting. During the formation of these cat's eyes, the nonlinear feedback of the higher order Fourier modes on the basic axisymmetric state, $\omega_{0}$, acts to flatten the vorticity profile around $r=r_{\text {ext }}$ producing an homogenized region of vorticity $[6,15]$. In reality this homogenized region often contains fine scale structures in nonlinear simulations, but the overall homogenization of $\omega_{0}$ around $r_{\text {ext }}$ is clear, for example see figure 3(a) in Turner \& Gilbert (2007) [15]. 
The response of the vortex to the external strain field with amplitude function (3.2) depends upon the nature of the critical layer which forms at $r_{\text {ext }}$. The width of the critical layer is $O\left(\widehat{A}^{1 / 2}\right)$ and it turns over on a time scale of $O\left(\widehat{A}^{-1 / 2}\right)$. Therefore, within the critical layer it is sensible to define the effective Reynolds number as

$$
R_{\text {eff }}=\widehat{A}^{3 / 2} R
$$

which is effectively the inverse of the Haberman parameter used in Le Dizès (2000) [14] and Haberman (1972) [11]. This definition also arises naturally in the critical layer analysis of Hall et al. (2003) [26].

When $R_{\text {eff }} \rightarrow \infty$ as $R \rightarrow \infty$ the critical layer is nonlinear in nature [10], but when $R_{\text {eff }} \rightarrow 0$ as $R \rightarrow \infty$ the critical layer is viscous in nature [13], with values in between giving a mixed effect. Le Dizès (2000) [14] showed that the nature of the critical layer can be compressed into the value of the phase jump of the perturbation streamfunction of the linear problem, $\chi$, as the solution crosses the critical layer. He showed that $\chi=0$ implies that nonlinearity dominates the critical radius and $\chi=-\pi$ implies that viscosity dominates at the critical radius.

For nonlinear critical layers Le Dizès (2000) [14] found one specific critical radius for strains with $n=2$ and $n=3$, at which infinitesimally thin, rotating cat's eyes can persist in a Gaussian votex without requiring an external strain field to maintain them. Thus, if infinitely thin cat's eyes were created by an external strain field at any radius other than this critical radius, they would decay away once the strain field was switched off. Turner \& Gilbert (2007) [15] showed that finite thickness cat's eyes can persist at other radii once the strain field is turned off, but in this case the basic vorticity profile was modified by the strain, hence the vortex is no longer Gaussian. The critical radius, which we denote by $r_{\text {cat }}^{\mathrm{NL}}$, has a corresponding angular velocity $\alpha_{\text {cat }}^{\mathrm{NL}}$ determined by solving $\alpha\left(r_{\text {cat }}^{\mathrm{NL}}\right)=\alpha_{\text {cat }}^{\mathrm{NL}}$. For $n=2$ and $n=3$ these values are found to be

$$
\begin{aligned}
& n=2 \quad r_{\text {cat }}^{\mathrm{NL}} \simeq 3.44, \quad \alpha_{\text {cat }}^{\mathrm{NL}} \simeq 0.0127, \\
& n=3 \quad r_{\text {cat }}^{\mathrm{NL}} \simeq 1.68, \quad \alpha_{\text {cat }}^{\mathrm{NL}} \simeq 0.0285,
\end{aligned}
$$

for the Gaussian vortex (2.3). For $n \geq 4$ Le Dizès (2000) [14] showed that no such radii exists in the Gaussian vortex. The consequence of these values is, if the strain field were tuned such that $r_{\text {ext }}=r_{\text {cat }}^{\mathrm{NL}}$ then we would expect a resonant response at this point and a rapid growth in the perturbation vorticity.

For viscous critical layers Le Dizès (2000) [14] found that no corresponding resonance radii exist for any $n$, but he did show that the maximum value of the non-axisymmetric perturbation vorticity (quasi-mode amplitude) is given by

$$
\omega_{\max }^{\mathrm{NA}}=B \widehat{A} R^{1 / 3} G_{n}(r),
$$

where $B$ is a numerical constant defined in Appendix A and $G_{n}(r)$ is given by (3.13) of Le Dizès (2000) [14], which is determined numerically. A plot of $G_{n}(r)$ is given in figure 2 for $n=2,3,4$. This figure is essentially a reproduction of figure 8 of Le Dizès (2000) [14] except we have plotted $G_{n}$ as a function of $r$ rather than $\alpha$, and our axisymmetric vortex is different, hence the $y$-axis is stretched. Despite no resonant radii existing for the viscous critical layer case, figure 2 shows that we should expect a large feedback response on $\omega_{0}(r, t)$ for radii close to the maximum value of $G_{n}(r)$ for each $n$. We denote the 


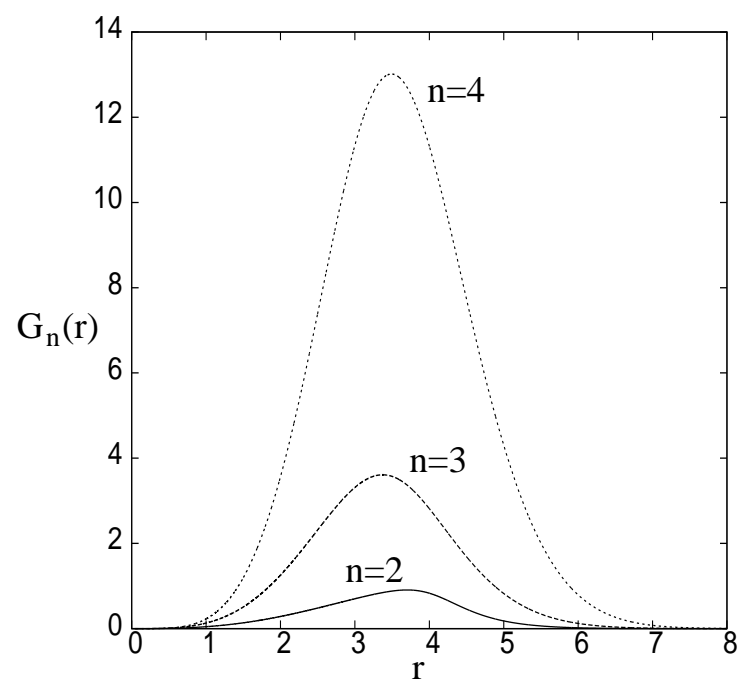

Figure 2: Plot of $G_{n}(r)$ from (3.4) for $n=2,3$ and 4 .

\begin{tabular}{ccc}
$n$ & $r_{\text {cat }}^{\mathrm{V}}$ & $\alpha\left(r_{\text {cat }}^{\mathrm{V}}\right)$ \\
\hline 2 & 3.705 & 0.0112 \\
3 & 3.375 & 0.0132 \\
4 & 3.496 & 0.0124 \\
\hline
\end{tabular}

Table 1: Table of values of $r$ at which $G_{n}(r)$ in figure 2 attains its maximum value, denoted by $r_{\text {cat }}^{\mathrm{V}}$, and the corresponding value of $\alpha\left(r_{\text {cat }}^{\mathrm{V}}\right)$.

value of $r$ at the maximum values of $G_{n}(r)$ by $r_{\text {cat }}^{\mathrm{V}}$ and table 1 gives these values and the corresponding angular velocities for $m=2,3$ and 4 .

In numerical simulations, the nonlinear limit of $R_{\text {eff }} \rightarrow \infty$ as $R \rightarrow \infty$ is hard to achieve because it requires very large Reynolds numbers or wide critical layers, and in this paper we are not able to achieve this nonlinear limit. In fact our simulations are run for weak strain fields (small amplitude), therefore we expect $R_{\text {eff }}$ to be moderately small, hence we expect staircases to form around the viscous critical layer values of $r_{\text {cat }}^{\mathrm{V}}$ in table 1. Note that this viscous critical layer limit was the limit considered by Turner et al. (2009) [17] and so the results presented here can be compared to their results.

In this article we consider the effect of a random strain field on the evolution of a vortex, which generates a number of viscous critical layers in the vortex. To implement this we use the procedure laid out by Kraichan (1970) [27] and Drummond et al. (1984) [28] and set

$$
q(t)=\phi(t) N_{F}^{-1 / 2} \sum_{j=1}^{N_{F}} \exp \left[-\mathrm{i}\left(n \zeta_{j} t+\nu_{j}\right)\right] .
$$

Here the coefficients $\zeta_{j}$ are chosen randomly from a normal distribution with mean $\mu$ and standard deviation $\sigma$ and $\nu_{j}$ are randomly chosen from a uniform distribution in the range $[0,2 \pi]$. The integer $n$ in $q(t)$ makes the $\zeta_{j}$ value the angular velocity of the $j^{\text {th }}$ component of the strain field. The function $\phi(t)$ merely acts as a switch which allows the random strain to be gradually switched on over a time $T$ to aid numerical implementation, 
and takes the form

$$
\phi(t)= \begin{cases}A \sin \left(\frac{\pi t}{2 T}\right) & \text { for } t<T \\ A & \text { for } t \geq T\end{cases}
$$

where $A$ is an amplitude parameter. The form of $q(t)$ in (3.5) leads to a smooth function comprising of $N_{F}$ strain field components each rotating with angular velocity $\zeta_{j}$. Each of these terms will act to flatten the azimuthally averaged vorticity profile $\omega_{0}(r, t)$ at a radius corresponding to the angular velocity $\zeta_{j}$. Due to the large feedback response on the vortex around $r_{\text {cat }}^{\mathrm{V}}$ we expect any flattening to be more pronounced in the vicinity of this radius. We also expect the strain field to interact with the modified azimuthally averaged profile producing a vorticity staircase similar to that observed in Turner et al. (2009) [17]. As $q(t)$ in (3.5) contains $N_{F}$ harmonics, then we can argue that this strain field leads to an effective Reynolds number approximately given by (3.3) with $\widehat{A}=N_{F}^{1 / 2} A$.

While the results presented in $\S 4$ can be compared to those in Turner et al. (2009) [17], there are expected to be some differences for the following reasons. Firstly, the results of Turner et al. (2009) [17] are ensemble averaged at the outset, whereas here the strain field is explicitly applied and the results are time averaged to remove the random strain fluctuations. Thus, as our simulations will generate finite time series, there is expected to be some minor dependence on the time-averaging method used. Also, the numerical scheme in Turner et al. (2009) [17] neglects higher order nonlinear terms and the evolution of the vortex profile is based on a weakly-nonlinear diffusion equation approach, which leads the authors to introduce a 'smoothing parameter' to smooth out the nonlinear processes. Such a parameter is not required in the current work, which is one of the strengths of the fully nonlinear simulations. The form of the numerical scheme in Turner et al. (2009) [17] essentially means their results are valid for inviscid vortices as $t \rightarrow \infty$ with $R_{\text {eff }} \rightarrow 0$. However, such results are difficult to construct in fully nonlinear simulations, and the added viscosity in this paper, for numerical stability, will affect the staircase evolution. Finally, Turner et al. (2009) [17] use a delta time correlated function $q(t)$, which (3.5) is not, however they show that other correlated functions also exhibit step structures, albeit with different step characteristics.

The external strain fields considered in this work are weak, i.e. the amplitude $A$ is not large enough to allow vortex stripping to occur [29]. In fact in the vicinity of the vortex the flows considered here are dominated by the rotational component of the flow which is generated by the vortex itself.

For the simulations presented in $\S 4$ we choose $N_{F}=1000$ and $T=1000$ in (3.5), and in order to compare the results of the various simulations we use the same realization of the random function $q(t)$ for all simulations, unless otherwise stated.

\section{Results}

In this section we present results of the nonlinear numerical simulations. The results are time averaged in order to average out the fluctuations from the random strain field so that they can be compared to those of Turner et al. (2009) [17]. The time averaging scheme used is a rolling average, such that the time-averaged, azimuthally-averaged, vorticity profile is given by

$$
\bar{\omega}_{0}(r, t)= \begin{cases}\frac{1}{t} \int_{0}^{t} \omega_{0}(r, t) d t & 0 \leq t<\tau \\ \frac{1}{\tau} \int_{t-\tau}^{t} \omega_{0}(r, t) d t & t \geq \tau\end{cases}
$$




\begin{tabular}{ccc}
$\mu$ & $r_{\text {ext }}$ at $\mu$ & $r_{\text {ext }}$ at $\mu \pm \sigma$ \\
\hline 0 & $\infty$ & {$[5.641, \infty]$} \\
0.009 & 4.178 & {$[3.249,6.308]$} \\
0.014 & 3.249 & {$[2.622,4.178]$} \\
0.028 & 1.731 & {$[1.244,2.208]$} \\
\hline
\end{tabular}

Table 2: Values of $\mu$ and the corresponding value of $r_{\text {ext }}$ at $\mu$ for the strain fields considered in $\S 4$. The standard deviation for each distribution is $\sigma=0.005$.

where the over bar signifies the time-averaged quantity. We choose $\tau=1000$ because this value gives clean time averaged results, without completely eliminating the fine structure of the random forcing. Other values of $\tau$ give similar results, but if $\tau$ is taken too large then the viscous spreading of the vortex will contaminate the results.

The results are presented for four different strain field parameter distributions. The mean value of the strain field angular velocity distribution, $\mu$, along with the corresponding value of $r_{\text {ext }}$ are given in table 2 for the four distributions. Each strain field has a standard deviation of $\sigma=0.005$. The reason for choosing these $\mu$ values is because for $\mu=0$ and $\mu=0.028$ the mean angular velocity value of the strain field lies away from the resonance feedback radii, while $\mu=0.009$ and $\mu=0.014$ force critical layers at radii close to these resonance feedback values. We expect the results for $\mu=0.009$ and 0.014 to give larger feedback responses and hence more clearly defined vorticity staircases then the $\mu=0$ and $\mu=0.028$ strain fields.

We first present results for the case when the azimuthal wavenumber $n=2$ in $\S 4.1$ before considering the effect of higher wavenumbers in $\S 4.2$.

\subsection{Strain fields with $n=2$}

For the case $n=2$, the theory in $\S 3$ shows that the largest viscous feedback between the vortex and the strain field occurs for strains with angular velocities $\alpha_{\text {ext }} \approx \alpha_{\text {cat }}^{\mathrm{V}}=0.0112$. Therefore, we expect the strains with $\mu=0.009$ and $\mu=0.014$ to have the largest feedback response, and potentially the most significant staircase structure. However, we note that the theory in $\S 3$, and in particular the values in table 1 , is only valid at early times, because as the strain field feeds back onto the profile $\omega_{0}(r, t)$ the position, and response amplitude, of $\omega_{\max }^{\mathrm{NA}}$ will change. The effect of this change is discussed in $\S 5$. In this section the strain field (3.5) is considered with amplitudes $A=2 \times 10^{-6}, 4 \times 10^{-6}$ and $6 \times 10^{-6}$ which correspond to $R_{\text {eff }} \approx 50.3,142.3$ and 261.4 respectively. Therefore, while these values of $R_{\text {eff }} \neq 0$, they are small enough to consider the nature of the critical layers as viscous rather than nonlinear.

The evolution of the vorticity value at the origin $\bar{\omega}_{\text {or }}(t)=\bar{\omega}_{0}(r=0, t)$ in figure 3 confirms that the largest feedback on $\bar{\omega}_{\text {or }}(t)$ occurs for the strains with $\mu=0.009$ and $\mu=0.014$ as predicted by the linear theory. For each forcing $\mu$ value we observe an initial region, $t \lesssim 2 \times 10^{4}$, where the nonlinear terms of (2.5) increase in magnitude and eventually saturate. In this region the vortex spreads more rapidly than the $O(R)$ spreading expected of a viscous vortex, however, beyond this time the decay rate is in accord with the $O(R)$ decay, as indicated by the top straight line. This rapid spreading of the vortex is caused by the azimuthally averaged vorticity profile $\bar{\omega}_{0}$ being modified in the 

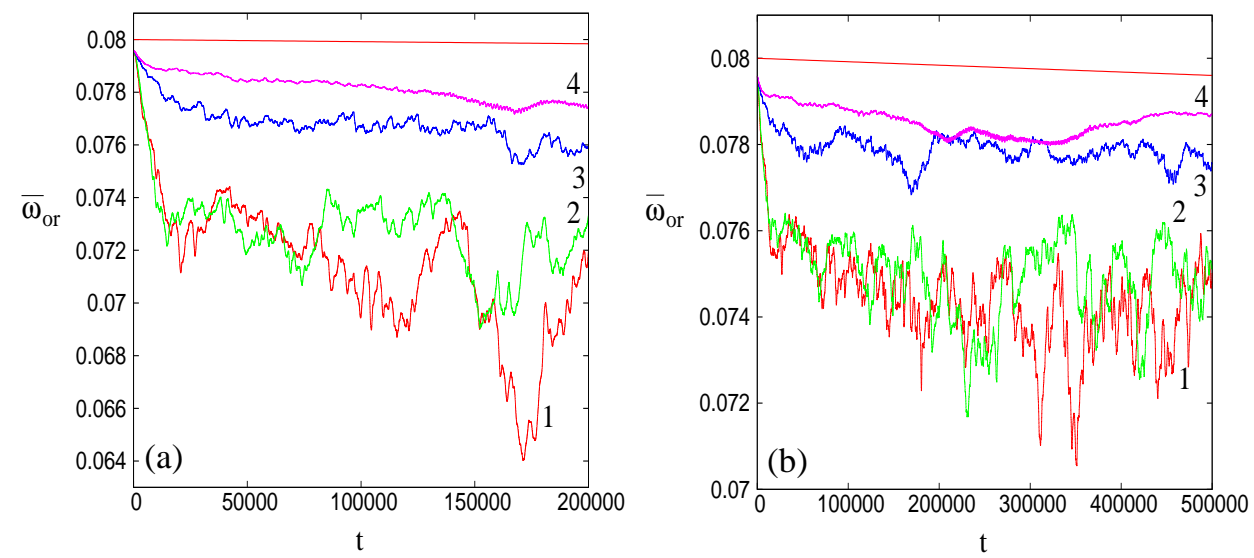

Figure 3: (colour online) Plot of $\bar{\omega}_{\text {or }}(t)=\bar{\omega}_{0}(0, t)$ for $R=10^{8}, n=2$ with (a) $A=$ $6 \times 10^{-6}$ and (b) $A=4 \times 10^{-6}$. In each panel the curves $\mu=0.009,0.014,0.0$ and 0.028 are numbered $1-4$ respectively. The top line has gradient $10^{-8}$ for comparison.

region close to $r_{\text {cat }}^{\mathrm{V}}$. Due to the conservation of angular momentum in the system, and the irrotational strain field not adding additional vorticity, the spreading of the vortex causes a spin down of the vortex at the core [16]. For the case $A=4 \times 10^{-6}$ in panel (b), we were able to run the simulations longer before the time stepping error contaminated the results, and thus these simulations are approaching the small forcing, large time simulations of Turner et al. (2009) [17].

The azimuthally averaged vorticity profiles $\bar{\omega}_{0}(r, t)$ for each of the results in figure $3(\mathrm{a})$ are given in figure 4 , with the time evolution reading down the curves from top to bottom. These plots all show the formulation of a vorticity staircase at the edge of the vortex. In panels (c) and (d) the staircases are less well defined due to the mean angular frequency of the strain being away from the largest feedback radii and thus $\omega_{\max }^{\mathrm{NA}}$ is smaller, so the modification of $\bar{\omega}_{0}$ is less. However, small steps are still visible in the final profiles. Therefore, irrespective of the mean angular velocity value, $\mu$, each strain field generates steps in approximately the same range of radial values. This range is approximately $r \in[2.5,4]$ which agrees well with the results of Turner et al. (2009) [17] where the staircases form approximately in $r \in[2.5,3.7]$.

For the results with $\mu=0.009$ and $\mu=0.014$ the staircases are more visible and form much faster than the other two strain fields. Also, in these cases the steps appear wider, but this could be because in panels (c) and (d) the steps are still forming. In each panel the steps drift slowly towards the origin over time. This drifting was also observed in Turner et al. (2009) [17] and the reason for this drift is due to the formation of the steps affecting the response of the vortex to the strain field. This is discussed in $\S 5$. One subtle difference between the results presented here and those in Turner et al. (2009) [17] is seen in figure 4(a). Here a sharp step forms during intermediate times and then disappears as the vorticity diffuses out from the edge of the vortex. However, the vortex does not form a sharp edge as in Turner et al. (2009) [17], but it does leave a large, well mixed, surf zone surrounding the remaining core. This can be seen in figure 5 .

An alternative way to visualize the time evolution of the vorticity profiles in figure 4 is to consider the space-time evolution of the vorticity gradient $-\left|\partial_{r} \bar{\omega}(r, t)\right|$ plotted in figure 5. These plots contain the same information as figure 4 , but instead show a continuous 

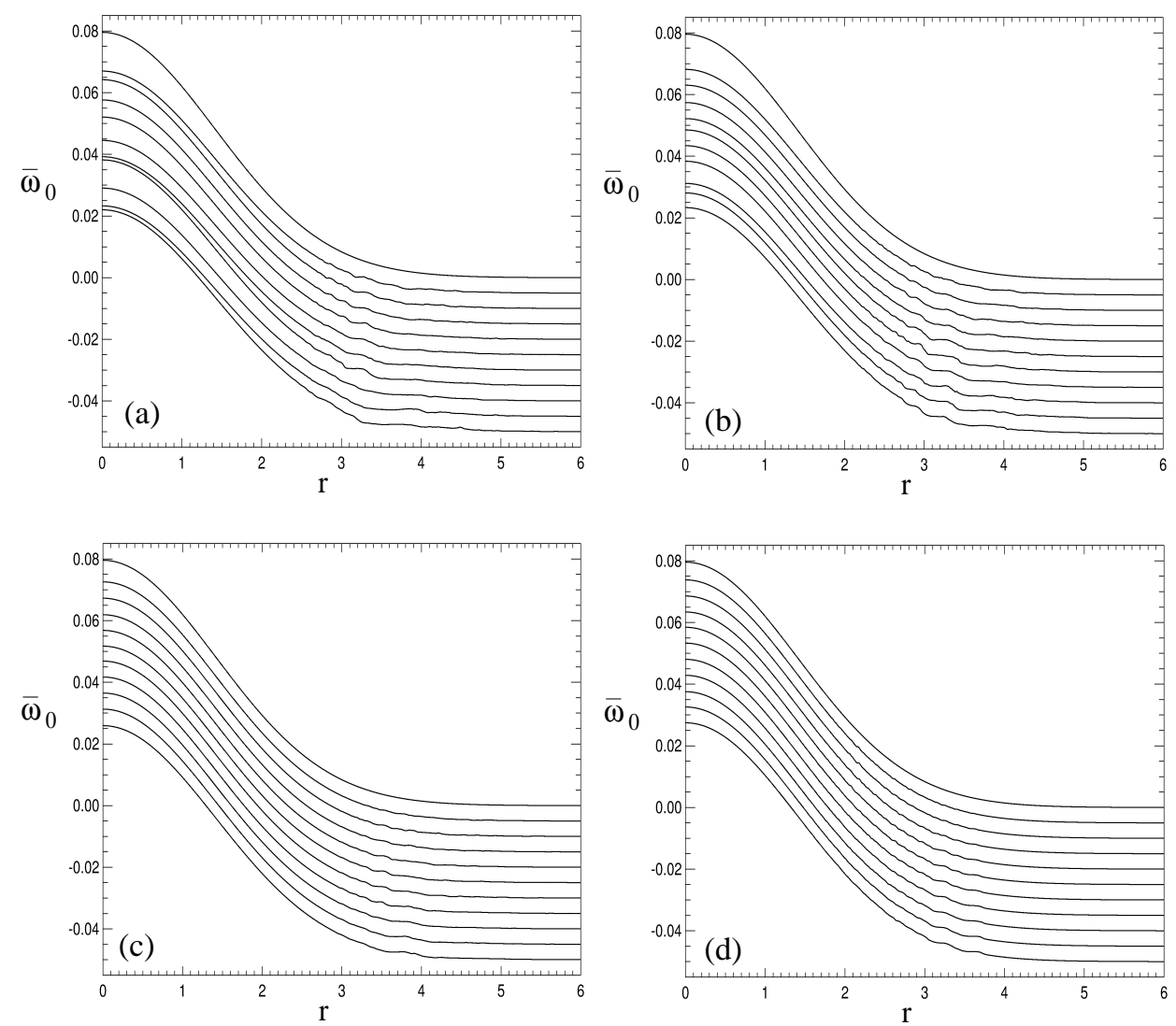

Figure 4: Profiles of $\bar{\omega}_{0}(r)$ for the results in figure 3(a). The panels correspond to (a) $\mu=0.009$, (b) 0.014, (c) 0.0 and (d) 0.028. In each panel the curves are separated by an additive constant and are given in steps of $t=2 \times 10^{4}$ reading down the curves.

evolution of the steps which form. These steps are indicated by the white regions, such as those around $r \approx 3$ in panels (a) and (b), and have steep edges indicated by grey regions. For the strain with $\mu=0.009$ in figure $5(\mathrm{a})$ we can see step merger taking place (see circled region), which was also observed in Turner et al. (2009) [17]. In this study we do not observe as many step merges as in Turner et al. (2009) [17], which is likely to be because the current simulations contain a small amount of viscosity which smooths out and removes small steps, reducing the number which form, and hence reducing the number of merges. What is clear from the plots in panels (a) and (b) are the surf zones outside the edge of the vortex where the vorticity is well mixed. In panels (c) and (d) the steps which form are much less pronounced, but these space-time figures clearly show the existence of these steps, and in fact, in both these cases the main steps which form appear to be at larger radii than in panels (a) and (b).

Using the linear theory of $\S 3$ we can predict an estimate of the initial range of radii over which steps are observed, given the strain parameters. In Turner \& Gilbert (2007) [15] it was observed that for the strain field to flatten the basic profile at a given radius the non-axisymmetric contribution of the vorticity field needed to be large enough to overcome the vorticity gradient of the basic profile. In the context of the linear theory 
5 (a)

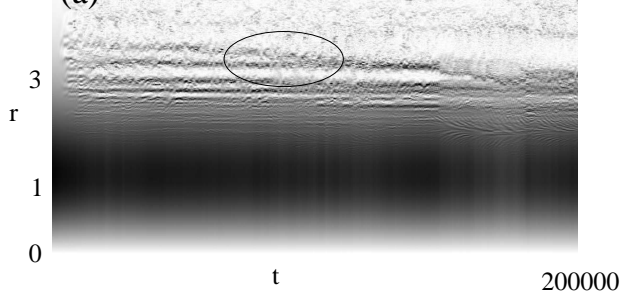

(c)
5 (b)

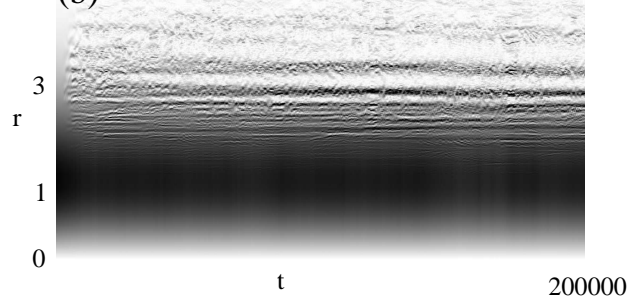

(d)

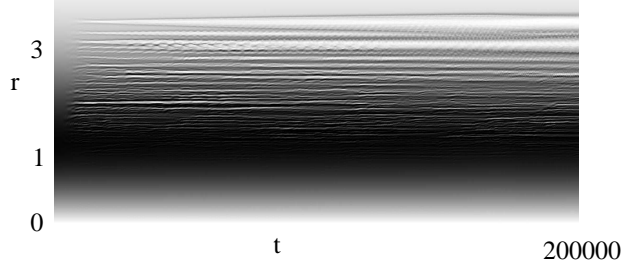

Figure 5: Space-time diagram of the evolution of the vorticity gradient - $\left|\partial_{r} \bar{\omega}(r, t)\right|$ plotted in grey scale in the $(t, r)$ plane for the results in figure 4 . The grey scale is capped at the level $-\left|\partial_{r} \bar{\omega}(r, t)\right|=-0.035$, corresponding to black; zero is white.

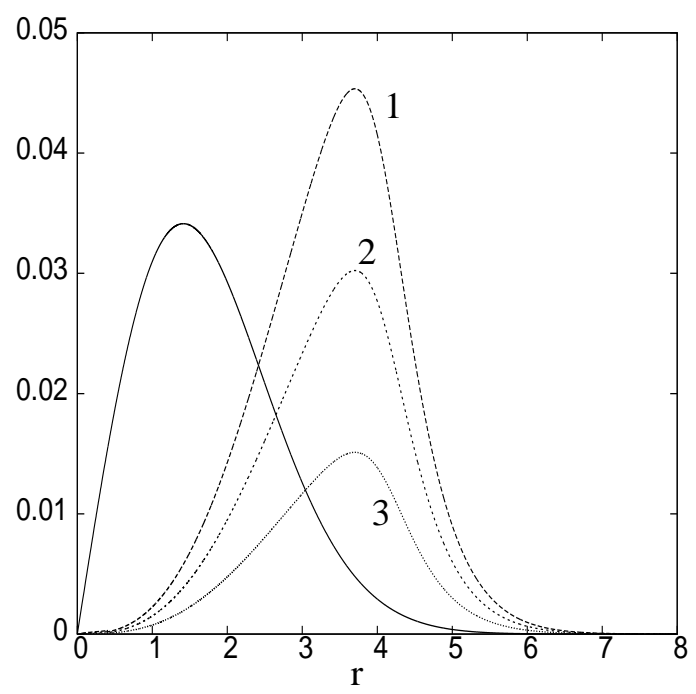

Figure 6: Plot of $C\left|\omega_{\max }^{\mathrm{NA}}\right|$ from (3.4) for $n=2$. The solid line represents $\left|\partial_{r} \Omega_{0}\right|$ while lines 1-3 denotes the results for $A=6 \times 10^{-6}, 4 \times 10^{-6}$ and $2 \times 10^{-6}$ respectively. The value of the combination $C B$ was chosen to be 0.568 so that line 1 crosses the solid curve at $r=r_{s} \approx 2.5$, the smallest radius at which steps were seen initially in figures 4 and 5 .

from $\S 3$ this means that the basic profile should flatten at radii for which

$$
C\left|\omega_{\max }^{\mathrm{NA}}\right| \geq\left|\partial_{r} \Omega_{0}\right|
$$

for some constant $C$. We observe in figures 4 and 5 that for $A=6 \times 10^{-6}$ steps initially form approximately for $r \in[2.5,4]$. In figure 6 we plot $C\left|\omega_{\max }^{\text {NA }}\right|$ with the combination $C B=0.568$ (curve 1 ) and the solid line depicts $\left|\partial_{r} \Omega_{0}\right|$. For this value of $C B$ the two curves cross at $r \approx 2.5$ and so $C\left|\omega_{\max }^{\mathrm{NA}}\right| \geq\left|\partial_{r} \Omega_{0}\right|$ for $r \gtrsim 2.5$. We denote this crossing 
point value where $C\left|\omega_{\max }^{\mathrm{NA}}\right|=\left|\partial_{r} \Omega_{0}\right|$ as $r_{s}$ and thus from (4.1) we only expect steps in the $A=6 \times 10^{-6}$ result for $r \gtrsim r_{s}$. This agrees with the observation in figures 4 and 5. Note that in figures 4 and 5 we do not see steps for $r \gtrsim 4$, but the above criteria predicts that we should observe steps in this range. The resolution of this comes from Turner \& Gilbert (2007) [15] who showed that forcing the Gaussian vortex (2.3) with a strain with $r_{\text {ext }} \gtrsim 4$ has little effect on the basic vorticity profile because the profile is already relatively flat at this point. Hence we do not expect to see steps in the Gaussian vortex (2.3) for $r \geq 4$, unless a significant amount of vorticity is advected from the core over time, increasing the vorticity gradient there.

The criteria (4.1) can now be used to predict staircase positions for different parameters. Results 2 and 3 in figure 6 show that $r_{s} \approx 2.7$ for $A=4 \times 10^{-6}$ and $r_{s}=3.0$ for $A=2 \times 10^{-6}$ respectively. These predictions agree very well with the results in figures 7 and 8 for $\mu=0.014$, strengthening the use of the prediction criteria (4.1).
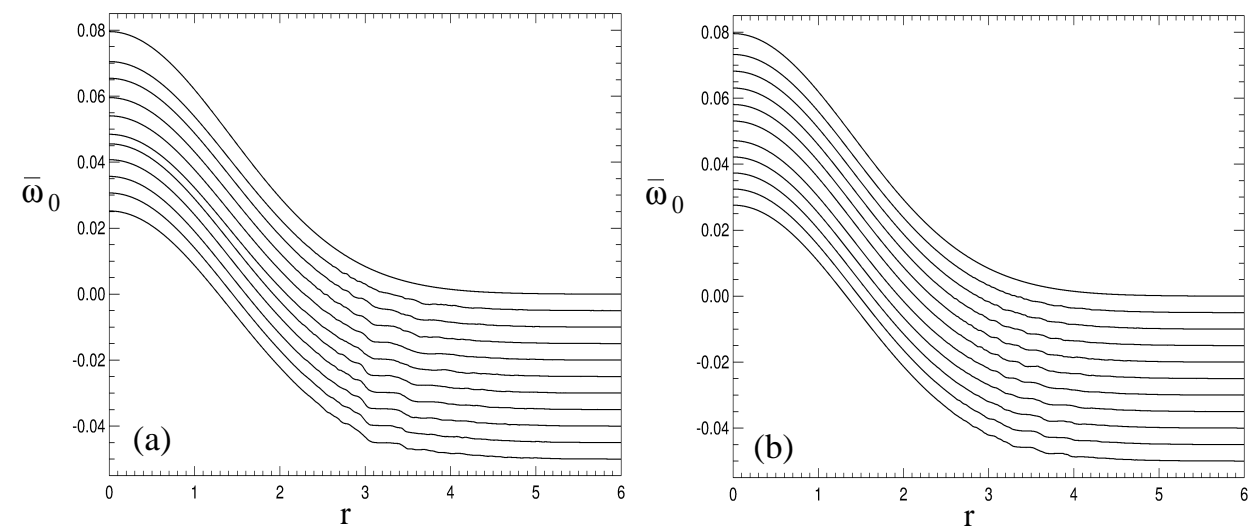

Figure 7: Profiles of $\bar{\omega}_{0}(r)$ with $\mu=0.014$ for (a) $A=4 \times 10^{-6}$ and (b) $A=2 \times 10^{-6}$. In each panel the curves are separated by an additive constant and are given in steps of $t=5 \times 10^{4}$ reading down the curves.

5 (a)

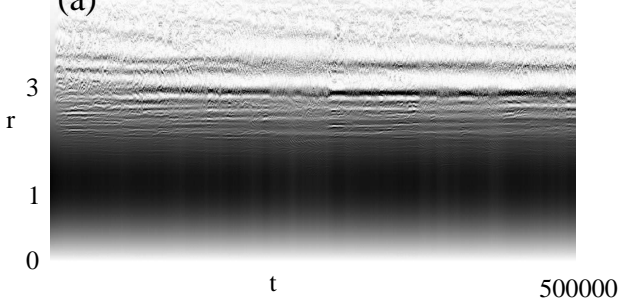

5 (b)

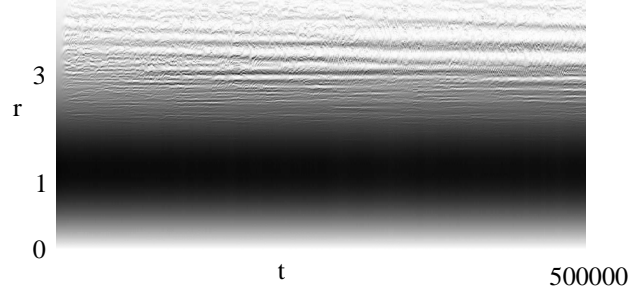

Figure 8: Space-time diagram of the evolution of the vorticity gradient - $\left|\partial_{r} \bar{\omega}(r, t)\right|$ plotted in grey scale in the $(t, r)$ plane for the results in figure 7 . The grey scale is capped at the level $-\left|\partial_{r} \bar{\omega}(r, t)\right|=-0.035$, corresponding to black; zero is white.

For the smaller amplitude profiles in figure 7 , we see that the $A=2 \times 10^{-6}$ result in panel (b) initially contains a lot of fine scale structure in the vorticity profile, but these slowly merge into two clear steps at the final time value, while for $A=4 \times 10^{-6}$ in panel 
(a), the small steps soon merge into one large step centred around $r \approx 3.25$. This merging of steps can be clearly seen in space-time plots in figure 8. Turner et al. (2009) [17] were not able to modify the amplitude of the nonlinear effects in a succinct manner such as reducing the forcing amplitude, but did so through varying their smoothing parameter. Just as we have found here, they identified that a small smoothing parameter (weak nonlinearity) produced more finer scale steps in the staircase which eventually merge over long time scales.

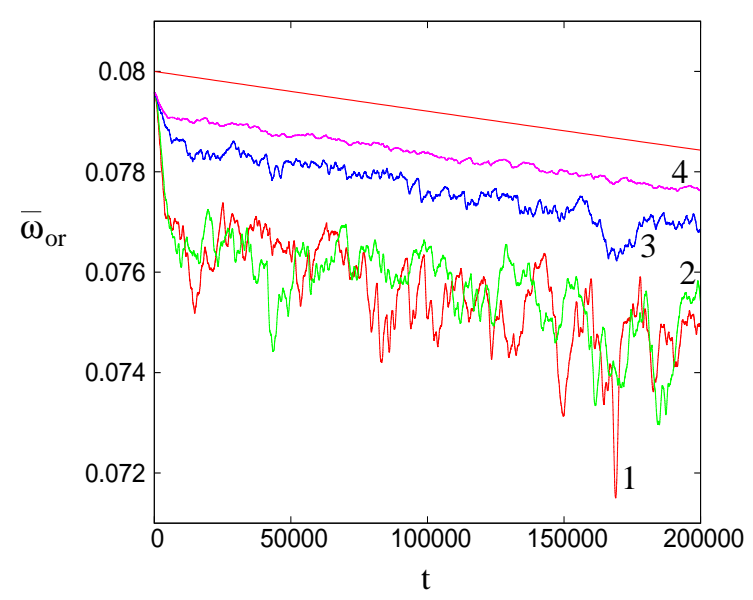

Figure 9: (colour online) Plot of $\bar{\omega}_{\text {or }}(t)$ for $R=10^{7}, n=2$ with $A=6 \times 10^{-6}$. In each panel the curves $\mu=0.009,0.014,0.0$ and 0.028 are numbered $1-4$ respectively. The top line has gradient $10^{-7}$ for comparison.

Increasing the viscosity within the vortex to $R=10^{7}$ has the effect of damping out the fine scale steps which form, as well as delaying the formation of the larger steps seen for $R=10^{8}$. It is worth noting that for $R=10^{7}$ the vortex now spreads considerably compared to the $R=10^{8}$ case, see figure 9 , where for all four results $\bar{\omega}_{\text {or }}(t)$ clearly decays at a rate proportional to $R$. We also observe from (3.4) that reducing $R$ by a factor of 10 reduces $\omega_{\max }^{\mathrm{NA}}$ by approximately a factor of 2 , so we expect the value of $r_{s}$ for $A=6 \times 10^{-6}$ to be similar to result 2 of figure 6 , and for $A=4 \times 10^{-4}$ the value to be similar to result 3 of figure 6.

Figures 10 and 11 plot the evolution of the $\mu=0.014$ result from figure 9 in panel (a) and the corresponding result with $A=4 \times 10^{-6}$ in panel (b). When we compare these results to the respective $R=10^{8}$ results we notice that the steps generated by the forcing are less well defined and narrower. In fact, for the smaller amplitude case in panel (b) the steps are hardly visible and appear to have disappeared by the final time point. However, the space-time diagrams in figure 11 do show the existence of steps, but the light grey regions surrounding them suggest that the steps are weak. In fact for $A=4 \times 10^{-6}$, no clear steps really emerges in figure 11(b) until around $t=2.5 \times 10^{4}$, which is much later than for the $R=10^{8}$ result. The increased viscosity acts to smooth out the sharp edges of the steps which eventually removes them from the periphery of the vortex altogether. The increased viscosity also causes the vortex to spread more rapidly than for $R=10^{8}$, and as it spreads the radii at which the external strain is acting moves towards the centre of the vortex where the vorticity gradient is larger. Hence the fixed value of $A$ is no longer large enough to flatten the vortex at these smaller radii and so the number of steps 

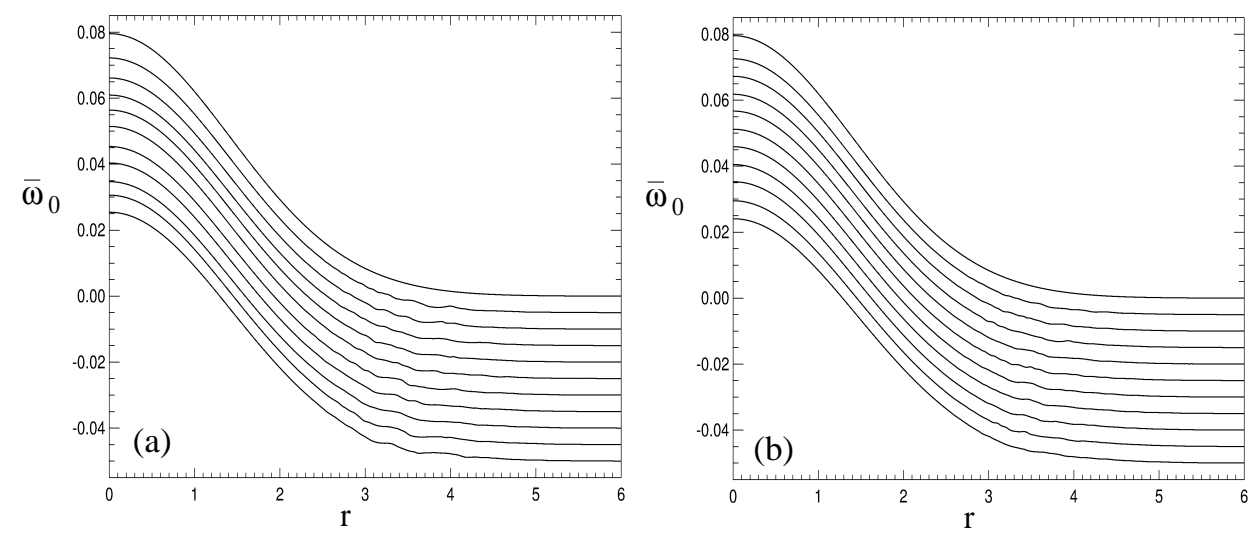

Figure 10: Profiles of $\bar{\omega}_{0}(r)$ for the $\mu=0.014$ result in figure 9. The panels correspond to (a) $A=6 \times 10^{-6}$ and (b) $A=4 \times 10^{-6}$. In each panel the curves are separated by an additive constant and are given in steps of (a) $t=2 \times 10^{4}$ and (b) $t=5 \times 10^{4}$ reading down the curves.

5 (a)

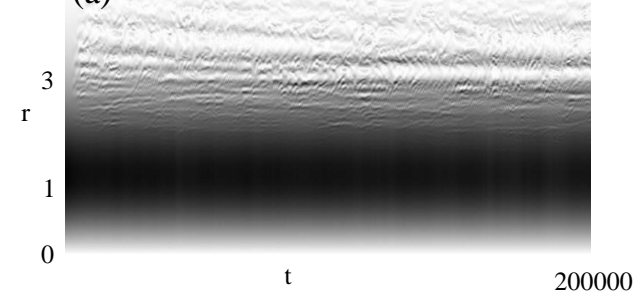

${ }^{5}(\mathrm{~b})$

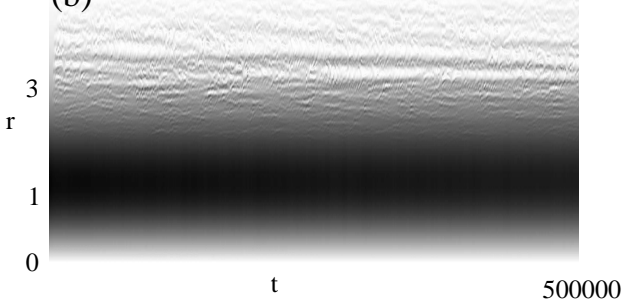

Figure 11: Space-time diagram of the evolution of the vorticity gradient - $\left|\partial_{r} \bar{\omega}(r, t)\right|$ plotted in grey scale in the $(t, r)$ plane for the results in figure 10. The grey scale is capped at the level $-\left|\partial_{r} \bar{\omega}(r, t)\right|=-0.035$, corresponding to black; zero is white.

is reduced. Note however, that the initial profiles show that the values of $r_{s}$ for these simulations predicted by (4.1) are in good agreement with the simulations, which can be more clearly seen in figure 11 .

The existence of the vorticity staircases in the vortex is independent of the random realization of the strain field, but the exact position, width and position of step mergers is affected by the realization, as seen in figure 12 . Here we plot results for $A=6 \times 10^{-6}$ with $\mu=0.009$, hence we compare the results in this figure with those in figure 4(a) and figure 5(a). While the initial step formation again occurs with $r_{s}=2.5$, the main long lasting step which forms centres around $r \approx 2.9$ for the second realization, compared to $r \approx 3.1$ for the first realization. However, we expect subtle variations in step position and width because firstly, the strain field is different and secondly the feedback onto $\omega_{0}$ is different which affects the position of the large feedback of the vortex, see $\S 5$. The key result here is, irrespective of the form of the random forcing, the staircase still forms initially with $r_{s} \approx 2.5$, and this staircase persists for large times. 

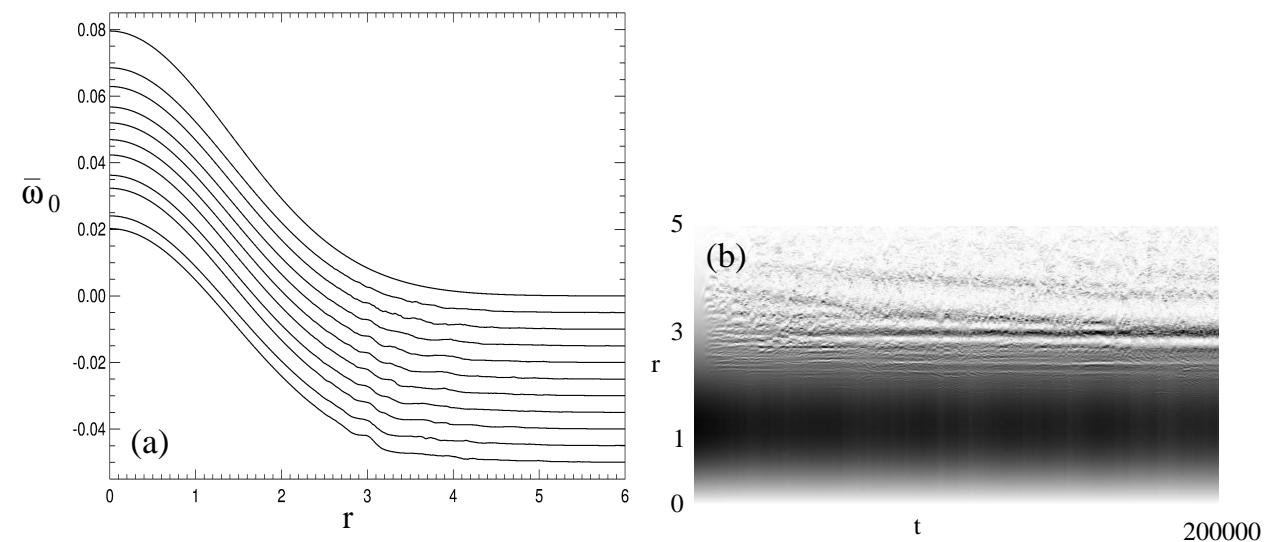

Figure 12: (a) Profiles of $\bar{\omega}_{0}(r)$ and (b) space-time diagram of the evolution of the vorticity gradient $-\left|\partial_{r} \bar{\omega}(r, t)\right|$ for the second realization of the random forcing with $\mu=0.009$ and $A=6 \times 10^{-6}$. In (a) the curves are separated by an additive constant and are given in steps of $t=2 \times 10^{4}$ reading down the curves.

\subsection{Strain fields with $n \geq 3$}

Here we consider strain fields with $n=3$ and $n=4$, and in this section we focus on the mean forcing frequencies closest to the viscous resonant values in table 1 , because these will give the largest feedback in the vortex, i.e. $\mu=0.009$ and 0.014 . Note that for these larger values of $n$ we have to use smaller amplitude values $A$ in order to stop the forcing from stripping off vorticity at large radii, and to make sure that the simulations remain independent of the discretization parameters. Thus for $n=3$ we use $A=2 \times 10^{-6}$ and for $n=4$ we use $A=0.25 \times 10^{-6}$ giving effective Reynolds numbers of $R_{\text {eff }}=50.3$ and 0.0125 respectively.

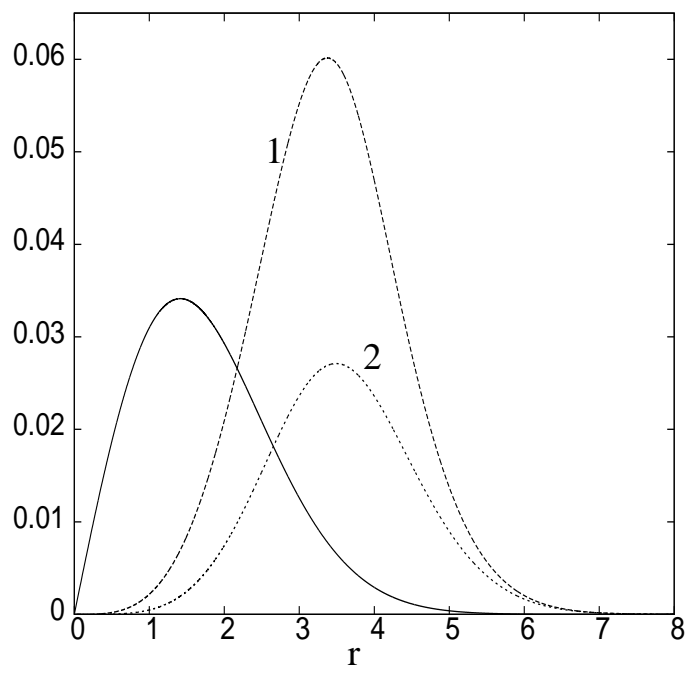

Figure 13: Plot of $C\left|\omega_{\max }^{\text {NA }}\right|$ from (3.4) for $n=3$ with $A=2 \times 10^{-6}$ and $n=4$ with $A=0.25 \times 10^{-6}$ represented by lines 1 and 2 respectively. The solid line represents $\left|\partial_{r} \Omega_{0}\right|$ and the value of the combination $C B=0.568$ is the same as in figure 6 . 
Figure 13 plots the inequality (4.1) for both $n=3$ and $n=4$ and we calculate that steps should be observed initially for $r \gtrsim r_{s}=2.2$ for $n=3$ and $r \gtrsim r_{s}=2.6$ for $n=4$. Thus for a larger value of $n$ with fixed $A$, we expect to see steps closer to the core of the vortex initially, because $r_{s}=3$ with $A=2 \times 10^{-6}$ and $n=2$ in $\S 4.1$.

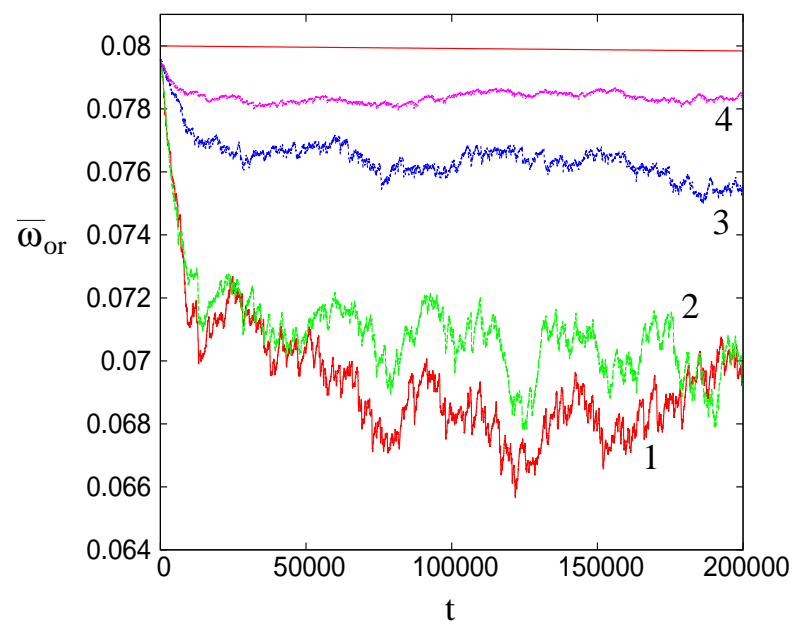

Figure 14: (colour online) Plot of $\bar{\omega}_{\text {or }}(t)=\bar{\omega}_{0}(0, t)$ for $R=10^{8}$ with $n=3$ and 4 and $A=2 \times 10^{-6}$ and $A=0.25 \times 10^{-6}$ respectively. The curves $\mu=0.009,0.014$ for $n=3$ are numbered 1 and 2 respectively while the curves for $\mu=0.009,0.014$ for $n=4$ are numbered 3 and 4 respectively. The top line has gradient $10^{-8}$ for comparison.

The results for $\bar{\omega}_{\text {or }}(t)$ in figure 14 show that for $n=3$ the response to the strain field is of a similar magnitude to that observed for $n=2$ in figure 3 . However, for $n=4$ the response is smaller, due to the fact that a smaller value of $A$ was used to avoid vortex stripping by the strain field. Therefore we might expect similar size steps to the $n=2$ results for $n=3$ and less well defined steps for $n=4$.

The azimuthally averaged vorticity profiles for the results in figure 14 are given in figure 15. The $n=3$ results in panels (a) and (b) show that the steps which form are narrower than those for $n=2$, and because of this, more steps form initially. This can more clearly be seen in the space-time plots in figures 16(a) and (b). These space-time plots also show that more step merges happen during the vortex evolution but at the finial time value of the simulations, there are typically only 1 or 2 larger steps. It should also be noted that the initial profiles in figures 15(a) and (b) show that steps only form for $r \gtrsim 2.2$ initially, which agrees with the prediction for $r_{s}$ from figure 13 .

Panels (c) and (d) of figures 15 and 16 show that when $n=4$ the steps which form are narrower still. In fact these results show that for this amplitude value, no coherent steps persist in the periphery of the vortex. The space-time plots show that steps do form initially, but they quickly merge with other steps and are eventually destroyed by the strain field and viscosity. Therefore, these results suggest that vorticity staircases are only likely to be observed for strain fields with azimuthal wavenumbers $n=2$ and $n=3$. Despite this, it is again clear from figure 16 that the fine scale structures which do form for $n=4$ do so initially for $r \gtrsim 2.8$ agreeing with the criteria (4.1). 

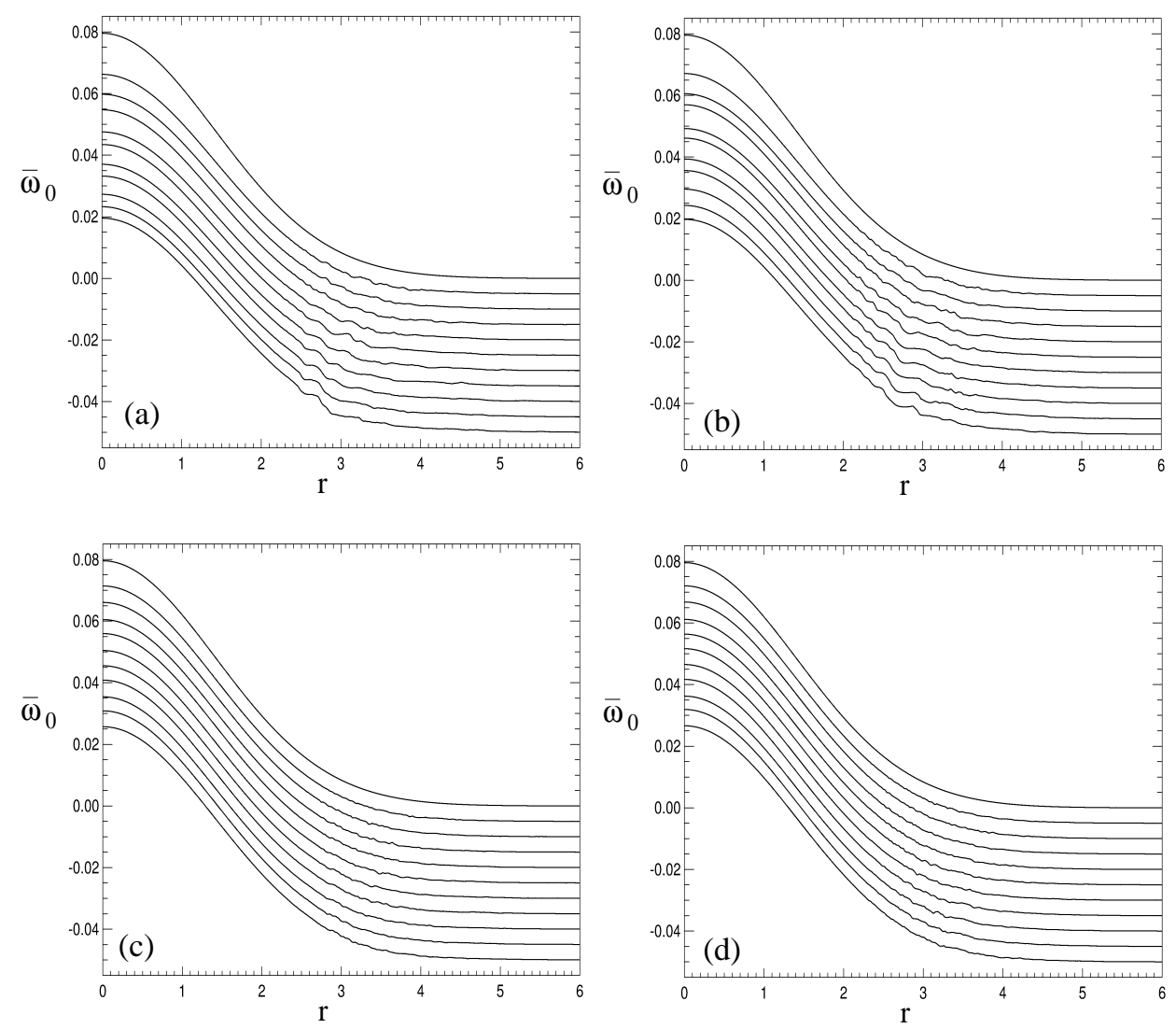

Figure 15: Profiles of $\bar{\omega}_{0}(r)$ for the results in figure 14. The panels correspond to (a) $(\mu, n)=(0.009,3)$, (b) $(0.014,3)$, (c) $(0.009,4)$ and (d) $(0.014,4)$. In each panel the curves are separated by an additive constant and are given in steps of $2 \times 10^{4}$ reading down the curves.

\section{Conclusions and Discussion}

This study examined the long time evolution of an axisymmetric Gaussian vortex in a weak-random-external strain field using fully nonlinear simulations. The results of this study show that for a multipolar strain field with azimuthal wavenumber $n$, a vorticity staircase forms at the periphery of the vortex. This staircase initially consists of many small steps which merge over time, typically into one or two larger steps. The results presented here reinforce the results presented in Turner et al. (2009) [17], who used a weakly-nonlinear analysis to derive a diffusion equation which was time-stepped to determine the evolution of the ensemble averaged vorticity profile, equivalent to our $\bar{\omega}_{0}(r, t)$. The results in Turner et al. (2009) [17] are essentially results for a very small amplitude strain field in the long time limit, which are difficult to replicate in numerical simulations. But the main drawback of their work was the need to introduce a 'smoothing' parameter to 'smooth' over the contribution of the nonlinear terms which were neglected in the diffusion equation approach. In the current paper we used simulations of the full Navier-Stokes equations, so the nonlinear terms were able to saturate and thus a smoothing parameter was not required. The results also showed that these steps act as barriers in the vortex, separating regions of well diffused vorticity from the coherent core of the vortex. This was 
5 (a)

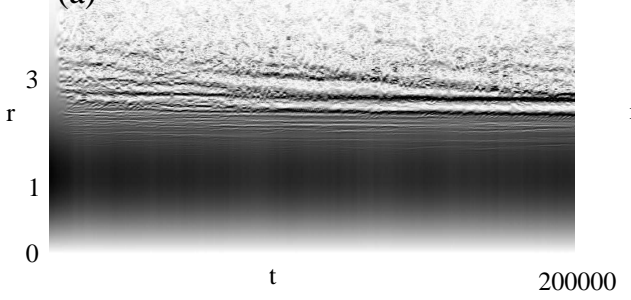

(c)

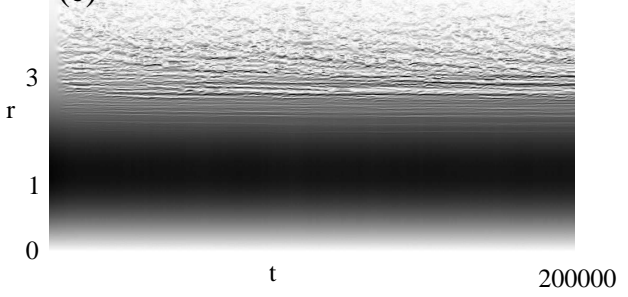

5 (b)

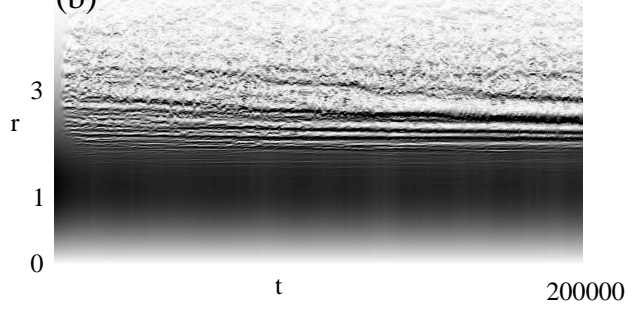

5 (d)

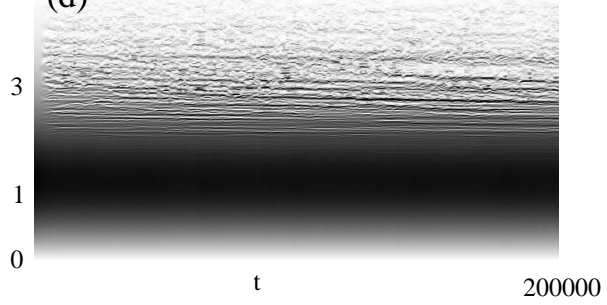

Figure 16: Space-time diagram of the evolution of the vorticity gradient - $\left|\partial_{r} \bar{\omega}(r, t)\right|$ plotted in grey scale in the $(t, r)$ plane for the results in figure 15. The grey scale is capped at the level $\left|\partial_{r} \bar{\omega}(r, t)\right|=0.035$, corresponding to black; zero is white.

clearly observed in the space-time diagrams where a clear, well mixed, surf zone existed outside the vortex, in most cases separated from the core by a single vorticity step.

The results in this paper showed that while the exact position and width of the persistent steps at later times depends on the strain parameters, the initial range of radii over which steps form was well predicted by the criteria (4.1). In $\S 4$ we used the results of our simulations to determine the value of the combination $C B=0.568$. Appendix $\mathrm{A}$ shows that $B \approx 1.288$, and hence the criteria (4.1) gives steps for radii where

$$
0.44\left|\omega_{\max }^{\mathrm{NA}}\right| \geq\left|\partial_{r} \Omega_{0}\right|
$$

It was found in the results in $\S 4$ that this criteria accurately predicted the initial step region for all the simulations.

The criteria (5.1) can also be used to predict the time evolution of the staircases by replacing $\Omega_{0}$ by the time averaged profile $\bar{\omega}_{0}$. To determine how flattening the vorticity profile affects the viscous critical layer feedback we consider (5.1) with $\Omega_{0}$ replaced by

$$
\bar{\omega}_{0}\left(r ; \epsilon, r_{\mathrm{c}}\right)=\frac{1}{4 \pi} e^{-r^{2} / 4}+\frac{r_{\mathrm{c}}\left(r-r_{\mathrm{c}}\right)}{8 \pi} \exp \left(\frac{r}{4 r_{\mathrm{c}}}\left(2-r_{\mathrm{c}}^{2}\right)-\frac{1}{2}-\frac{\left(r-r_{\mathrm{c}}\right)^{2}}{\epsilon^{2}}\right),
$$

which is the Gaussian vortex (2.3) with a flat region of width $\epsilon$ centred at $r=r_{c}$ (see Turner et al. (2008) [30]). Figure 17(a) shows the equality (5.1) for this profile with $r_{c}=3.0$ and $\epsilon=0.2$. This figure shows that when a flat region is formed in the vortex, the critical layer response at the edge of the steps is increased, particularly on the side of the step closer to the origin. This increased feedback leads to further flattening of the vortex on this side of the step which causes the step to drift towards the core of the vortex. This explains the drifting of the steps as seen in this article, such as figure 4, and in the work of Turner et al. (2009) [17].

The Gaussian vortex (2.3) is not always typical of vortices observed in physical situations. For example, vortices observed in two-dimensional turbulence often have sharp 

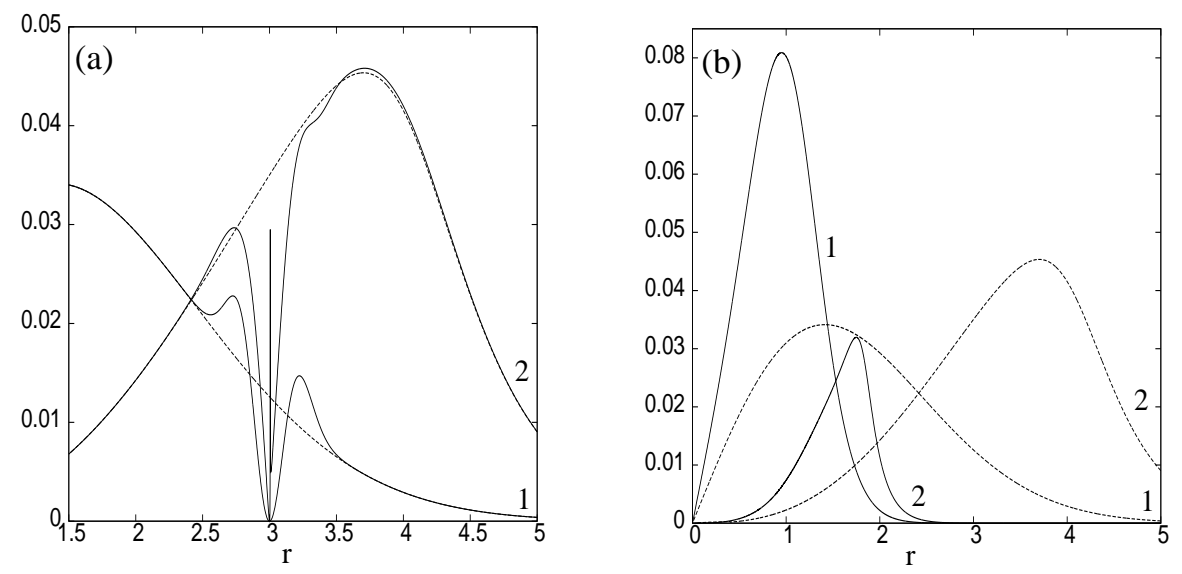

Figure 17: Plot of $\left|\partial_{r} \bar{\omega}_{0}\right|$ (curves 1) and $C\left|\omega_{\max }^{\mathrm{NA}}\right|$ (curves 2) for the vorticity profiles given in (a) (5.2) with $\left(r_{c}, \epsilon\right)=(3.0,0.2)$ and (b) (5.3) with $\sigma_{t}=0.7$ given by the solid curves. In both panels the results use $R=10^{8}, A=6 \times 10^{-6}$ and the dashed curves give the corresponding result for the Gaussian vortex (2.3) given in figure 6.

edges due to processes such as vortex stripping [29]. We can also consider how the criteria (5.1) depends upon these sharper edge vortices by considering the family of tanh vortices used by Hall et al. (2003) [26] and Turner \& Gilbert (2007) [15] with

$$
\bar{\omega}_{0}(r)=\frac{1}{4 \pi} \frac{1-\tanh \left[\left(r^{2}-\sigma_{t}^{2}\right) / 4\left(1-\sigma_{t}\right)\right]}{1+\tanh \left[\sigma_{t}^{2} / 4\left(1-\sigma_{t}\right)\right]}
$$

where $\sigma_{t} \in[0,1)$, and the vortex approaches a Rankine vortex as $\sigma_{t} \rightarrow 1$. The viscous resonant feedback response for this vortex with $\sigma_{t}=0.7$ in figure 17(b) shows that the region over which steps are expected to form reduces dramatically for sharp edged vortices. This suggests that in sharper edge vortices the formation of vorticity staircases maybe more difficult, and if they do form, the region in which they are generated will be small.

\section{- Appendix -}

\section{A Value of constant B in (3.4)}

The constant $B$ in (3.4) is given in the appendix of Le Dizès (2000) [14] as

$$
B=\max _{\{s \text { real }\}}\left|\operatorname{Re}\left(\bar{\omega}_{3}\right)\right|,
$$

where $\bar{\omega}_{3}(s, t)$ satisfies the viscous critical layer equation

$$
\frac{\partial^{2} \bar{\omega}_{3}}{\partial s^{2}}-s \bar{\omega}_{3}=1
$$

with

$$
\bar{\omega}_{3} \sim-\frac{1}{s} \quad \text { as } \quad s \rightarrow \pm \infty
$$




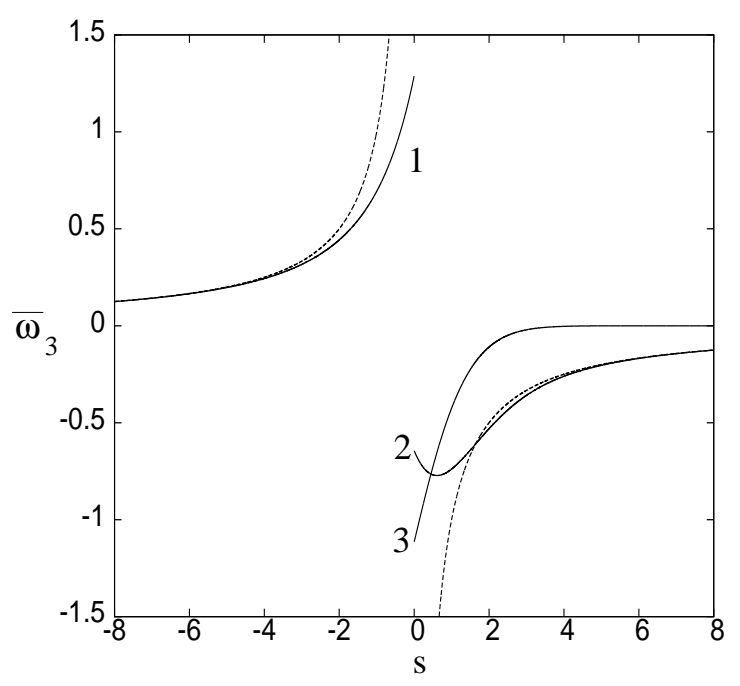

Figure 18: Plot of $\bar{\omega}_{3}(s)$. Curves 1 and 2 correspond to the real part, 3 gives the imaginary part, and the dashed lines give the large $s$ asymptotic behaviour.

The solution to this problem can be found in the appendix of Drazin \& Reid (1981) [31] and is

$\bar{\omega}_{3}=\left\{\begin{array}{cc}\pi\left[\frac{2}{3} \operatorname{Bi}(s)+\operatorname{Bi}(s) \int_{0}^{s} \operatorname{Ai}(\xi) d \xi-\operatorname{Ai}(s) \int_{0}^{s} \operatorname{Bi}(\xi) d \xi\right] & s \leq 0 \\ \pi e^{-2 \pi \mathrm{i} / 3}\left[\frac{2}{3} \operatorname{Bi}\left(s e^{-2 \pi \mathrm{i} / 3}\right)+\operatorname{Bi}\left(s e^{-2 \pi \mathrm{i} / 3}\right) \int_{0}^{s e^{-2 \pi \mathrm{i} / 3}} \operatorname{Ai}(\xi) d \xi-\operatorname{Ai}\left(s e^{-2 \pi \mathrm{i} / 3}\right) \int_{0}^{s e^{-2 \pi \mathrm{i} / 3}} \operatorname{Bi}(\xi) d \xi\right] & s \geq 0\end{array}\right.$

where $\mathrm{Ai}$ and $\mathrm{Bi}$ are Airy functions of the first kind. This solution is plotted in figure 18 with the corresponding large $s$ asymptotic behaviour. It is clear that the maximum value of the real part occurs at $s=0^{-}$and hence

$$
B=\frac{2}{3} \pi \operatorname{Bi}(0) \approx 1.288
$$

\section{References}

[1] L. M. Polvani and R. A. Plumb, "Rossby wave breaking, microbreaking, filamentation, and secondary vortex formation: the dynamics of a perturbed vortex," $J$. Atmospheric Sci. 49(6), 462 (1992).

[2] J. Thuburn and V. Lagneau, "Eulerian mean, contour integral, and finite-amplitude wave activity diagnostics applied to a single-layer model of the winter Stratosphere," J. Atmospheric Sci. 56(5), 689 (1999).

[3] H. K. Moffatt, S. Kida and K. Ohkitani, "Stretched vortices - the sinews of turbulence; large-Reynolds-number asymptotics," J. Fluid Mech 259, 241 (1994).

[4] J. Jiménez, H. K. Moffatt and C. Vasco, "The structure of the vortices in freely decaying two-dimensional turbulence," J. Fluid Mech. 313, 209 (1996).

[5] A. P. Bassom and A. D. Gilbert, "The spiral wind-up of vorticity in an inviscid planar vortex," J. Fluid Mech. 371, 109 (1998). 
[6] A. P. Bassom and A. D. Gilbert, "The spiral wind-up and dissipation of vorticity and a passive scalar in a strained planar vortex." J. Fluid Mech. 398, 245 (1999).

[7] D. A. Schecter, D. H. E. Dubin, A. C. Cass, C. F. Driscoll, I. M. Lansky and T. M. O'Neil, "Inviscid damping of asymmetries on a two-dimensional vortex," Phys. Fluids 12(10), 2397 (2000).

[8] N. J. Balmforth, S. G. Llewellyn Smith and W. R. Young, "Disturbing Vortices," J. Fluid Mech. 426, 95 (2001).

[9] R. J. Briggs, J. D. Daugherty and R. H. Levy, "Role of Landau damping in crossedfield electron beams and inviscid shear flow," Phys. Fluids 13, 421 (1970).

[10] D. J. Benney and R. F. Bergeron, "A new class of nonlinear waves in parallel flows," Stud. Appl. Maths 48, 181 (1969).

[11] R. Haberman, "Critical layers in parallel flows," Stud. Appl. Maths 51, 139 (1972).

[12] F. T. Smith and R. J. Bodonyi, "Nonlinear critical layers and their development in streaming-flow stability," J. Fluid Mech. 118, 165 (1982).

[13] C. C. Lin, "The Theory of Hydrodynamics Stability," Cambridge University Press (Cambridge) (1955).

[14] S. Le Dizès, "Non-axisymmetric vortices in two-dimensional flows," J. Fluid Mech. 406, 175 (2000).

[15] M. R. Turner and A. D. Gilbert, "Linear and nonlinear decay of cat's eyes in twodimensional vortices, and the link to Landau poles," J. Fluid Mech. 593, 255 (2007).

[16] M. R. Turner and A. D. Gilbert, "Spreading of two-dimensional axisymmetric vortices exposed to a rotating strain field," J. Fluid Mech. 630, 155 (2009).

[17] M. R. Turner, A. P. Bassom and A. D. Gilbert, "Diffusion and the formation of vorticity staircases in randomly strained two-dimensional vortices," J. Fluid Mech. 638, 49 (2009).

[18] M. E. McIntyre, "How well do we understand the dynamics of stratospheric warmings?," J. Meteorol. Soc. Japan 60, 37 (1982).

[19] D. G. Dritschel and M. E. McIntyre, "Multiple jets as PV staircases: the Philips effect and the resilience of eddy-transport barriers," J. Atmos. Sci. 65, 855 (2008).

[20] T. J. Dunkerton and R. K. Scott, "A barotropic model of the angular momentum conserving potential vorticity staricase in spherical geometry," J. Atmos. Sci. 65, 1105 (2008).

[21] R. K. Scott and D. G. Dritschel, "The structure of zonal jets in geostrophic turbulence," J. Fluid Mech. 711, 576 (2012).

[22] H. K. Moffatt and H. Kamkar, "On the time-scale associated with flux expulsion. In: Stellar and Planetary Magnetism (Ed. A. M. Soward)," Gordon \& Breach Science Publishers, 91 (1983). 
[23] P. B. Rhines and W. R. Young, "How rapidly is a passive scalar mixed within closed streamlines?," J. Fluid Mech. 133, 133 (1983).

[24] A. J. Bernoff and J. F. Lingevitch, "Rapid relaxation of an axisymmetric vortex," Phys. Fluids 6, 3717 (1994).

[25] K. Bajer, A. P. Bassom and A. D. Gilbert, "Accelerated diffusion in the centre of a vortex," J. Fluid Mech. 437, 395 (2001).

[26] I. M. Hall, A. P. Bassom and A. D. Gilbert, "The effect of fine structure on the stability of planar vortices," Eur. J. Mech. B Fluids 22(2), 179 (2003).

[27] R. H. Kraichan, "Diffusion by a random velocity field," Phys. Fluids 13, 22 (1970).

[28] I. T. Drummond, S. Duane and R. R. Horgan, "Scalar diffusion in simulated helical turbulence with molecular diffusivity," J. Fluid Mech. 138, 75 (1984).

[29] B. Legras and D. G. Dritschel, "Vortex stripping and the generation of high vorticity gradients in two-dimensional flows," Appl. Sci. Res. 51, 445 (1993).

[30] M. R. Turner, A. D. Gilbert and A. P. Bassom, "Neutral modes of a two-dimensional vortex and their link to persistent cat's eyes," Phys. Fluids 20(2), 027101 (2008).

[31] P. G. Drazin and W. H. Reid, Hydrodynamic Stability. Cambridge University Press (Cambridge), (1981). 